\title{
Lenticulostriate Artery Aneurysm Presenting as Subarachnoid Haemorrhage
}

Trilochan Srivastava, Raghavendra Bakki Sannegowda*, Bhawna Sharma, Tarun Mathur and Borislav Atzev

Department of Neurology, SMS Medical College Hospital, Jaipur, Rajasthan, India

Keywords: Lenticulostriate artery aneurysm; Subarachnoid hemorrhages; Intraventricualr hemmorrhage

\section{Introduction}

Lenticulostriate perforating artery aneurysms are rare [1] Lenticulostriate artery (LSA) aneurysms are endarteries, small and fragile and supply internal capsule and basal ganglia and LSA aneurysms may be saccular or fusiform and commonly present with basal ganglia hemorrhage $[1,2]$. They can be associated with conditions like moyamoya disease, hypertension, vasculitis and arterio-venous malformations (AVMs) [1-4]. LSA aneurysms which by itself is an uncommon entity usually presents with intraparenchymal haemorrhages [3,5]. Rarely LSA aneurysms presents with subarachnoid haemorrhage $(\mathrm{SAH})$ or primary intraventricular haemorrhage PIVH $[1-3,5,6]$. We report a rare case of LSA aneurysm presenting with SAH who was successfully managed conservatively.

\section{Case Report}

We present a 60 year old female without any history of comorbid illness presented with sudden onset severe headache followed by altered sensorium and vomiting. There was no history of seizures or head injury. On examination her Glasgow coma scale (GCS) was 12 and had left hemiparesis (power of 2/5). Emergency non contrast CT head revealed SAH (Figure 1a), she was stabilized with symptomatic treatment with adequate hydration. Tablet nimodepine $60 \mathrm{mg}$ was given every $4^{\text {th }}$ hourly through nasogastric tube to prevent any vasospasm secondary to SAH as patient had already developed hemiparesis though there were no demonstrable infarcts on CT. After initial stabilization, CT angio was done which revealed right proximal lenticulostriate artery (LSA) aneurysm (Figure 1b). Catheter angiography in anterio-posterior (Figure 1c) and lateral view (Figure 1d) confirmed saccular type of right proximal LSA aneurysm though there were no demonstrable vasospasms. Since patient family members were not willing for any active intervention she was treated conservatively with hydration and nimodipine, with gradual recovery in her sensorium over next 10 days. 3 weeks later she was discharged with normal sensorium and with mild improvement in her power (of 3/5). One of the limitation of this study is that we could not get follow-up of this patient to assess her clinical outcome as well as to do repeat cerebral angiography to know the status of LSA aneurysm.

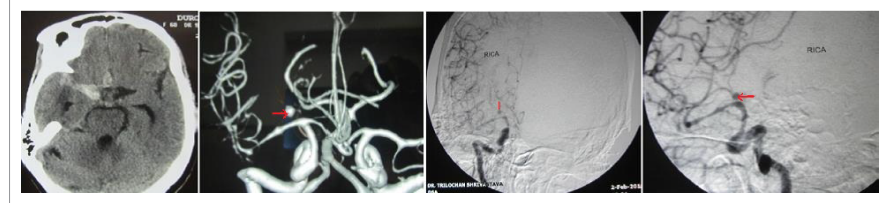

Figure 1: 1a: Plain CT head showing subarachnoid hemorrhage in right perisylvian region and interhemispheric fissure with ventricular dilatation, 1b: CT angiogram brain showing saccular aneurysm (arrow) arising from right proximal lateral lenticulostriate artery, branch of middle cerebral artery, 1c: Catheter angiography (right ICA injection) anterio-posterior view and 1d (lateral view) showing right proximal lateral lenticulostriate artery aneurysm (arrow).

\section{Discussion}

Around 29 cases of aneurysm arising from LSA have been reported $[2,3]$. Most are located in the distal course of the artery, within the basal ganglia. Proximal LSA aneurysms are most often middle cerebral artery (MCA) -LSA junction aneurysms, involving some part of the MCA trunk [4]. They are usually associated with conditions like moyamoya disease, hypertension, vasculitis and AVMs [2]. LSA aneurysms may be saccular or fusiform and usually presents with intraparenchymal haemorrhage and commonly with basal ganglia haemorrhage [2]. Other than deep intraparenchymal hemorrhage, LSA aneurysms can also present in various types either in isolation or in combination such as subarachnoid hemorrhage (SAH), intraventricular hemorrhage (IVH) [6]. LSA aneurysm rupture can present with isolated intraventricular haemorrhage and must be given diagnostic consideration in cases of PIVH [7]. Rarely LSA aneurysm can present as isolated subarachnoid hemorrhage (SAH) [1-3]. Eddlemen et al. [1] and Bhat et al. [2] reported a case of pure SAH due to rupture of the proximal LSA fusiform aneurysm. Kochar et al. [3] described a rare case of ruptured fusiform LSA aneurysm presenting as SAH alone, arising from proximal M2 MCA branch, which was detected on superselective microcatheter angiography.

LSA aneurysms may not be detected on initial angiograms because of their small size $(5 \mathrm{~mm})$ and deep location ${ }^{6}$. Catheter cerebral angiography should be considered when noninvasive imaging is unrevealing $[7,8]$.

Treatment of LSA aneurysms is still a controversy because of small patient numbers [6]. Because of the relatively inaccessible location of these lesions for direct surgical or endovascular occlusion, conservative management may be a therapeutic option in patients with LSA aneurysm, and spontaneous resolution can be observed [7]. Conservative approach involving close angiographic monitoring may be proposed as first-line treatment. If the monitored aneurysm then persists or grows in size, its occlusion should be considered. However studies are needed to strengthen this strategy [9]. Definitive treatment of LSA aneurysms involves both surgical and endovascular methods $[3,8]$. Tsai et al. [10] reported a case of LSA aneurysm that was successfully embolized with n-butyl cyanoacrylate (n-BCA) with no recurrence after 1 year of follow-up.

${ }^{*}$ Corresponding author: Raghavendra Bakki Sannegowda, Assistant Professor Department of Neurology, Father Muller Medical College, Karnataka, India, Tel: 9986144799; E-mail: padayappa4u@gmail.com

Received March 11, 2014; Accepted May 10, 2014; Published May 12, 2014

Citation: Srivastava T, Sannegowda RB, Sharma B, Mathur T, Atzev (2014) Lenticulostriate Artery Aneurysm Presenting as Subarachnoid Haemorrhage. J Vasc Med Surg 2: 138. doi: 10.4172/2329-6925.1000138

Copyright: (c) 2014 Srivastava T, et al. This is an open-access article distributed under the terms of the Creative Commons Attribution License, which permits unrestricted use, distribution, and reproduction in any medium, provided the original author and source are credited. 
Citation: Srivastava T, Sannegowda RB, Sharma B, Mathur T, Atzev (2014) Lenticulostriate Artery Aneurysm Presenting as Subarachnoid Haemorrhage. J Vasc Med Surg 2: 138. doi: 10.4172/2329-6925.1000138

Page 2 of 2

To conclude LSA aneurysms are as such rare and presenting as SAH is further a rare entity. Catheter angiography may be helpful in patients with SAH to identify these rare etiological causes.

\section{References}

1. Eddleman CS, Surdell D, Pollock G, Batjer HH, Bendok BR (2007) Ruptured proximal lenticulostriate artery fusiform presenting with subarachnoid hemorrhage: case report. Neurosurgery 60: E949.

2. Bhat DI, Shukla DP, Somanna S (2012) Subarachnoid hemorrhage from a ruptured proximal lenticulostriate artery aneurysm. Neurol India 60: 128-129.

3. Kochar PS, Morrish WF, Hudon ME, Wong JH, Goyal M (2010) Fusiform lenticulostriate artery aneurysm with subarachnoid hemorrhage: The role for superselective angiography in treatment planning. Interv Neuroradiol 16: 259263.

4. Vates GE, Arthur KA, Ojemann SG, Williams F, Lawton MT (2001) A neurocytoma and an associated lenticulostriate artery aneurysm presenting with intraventricular hemorrhage: Case report. Neurosurgery 49: 721-725.
5. Srivastava T, Sannegowda RB, Sharma B, Tejwani S (2013) Lenticulostriate artery aneurysm presenting as primary intraventricular haemorrhage. BMJ case reports.

6. Ahn JY, Cho JH, Lee JW (2007) Distal lenticulostriate artery aneurysm in deep intracerebralhaemorrhage. J Neurol Neurosurg Psychiatry 78: 1401-1403.

7. Ellis JA, D'Amico R, Altschul D, Leung R, Connolly ES, et al. (2011) Medial lenticulostriate artery aneurysm presenting with isolated intraventricular hemorrhage. Surg Neurol Int 2: 92.

8. Larrazabal R, Pelz D, Findlay JM (2001) Endovascular treatment of a lenticulostriate artery aneurysm with $\mathrm{N}$-butyl cyanoacrylate. Can J Neurol Sci 28: 256-259.

9. Heck O, Anxionnat R, Lacour JC, Derelle AL, Ducrocq X, et al. (2014) Rupture of lenticulostriate artery aneurysms. J Neurosurg 120: 426-433.

10. Tsai YH, Wang TC, Weng HH, Wong HF (2011) Embolization of a ruptured lenticulostriate artery aneurysm. J Neuroradiol 38: 242-225. 